\title{
IS PLATELET TO MONOCYTE RATIO A USEFUL INFLAMMATORY DETERMINANT OF ST-ELEVATION MYOCARDIAL INFARCTION?
}

\author{
İbrahim Çaltekin ${ }^{\top} \oplus$, Erdal Demirtaş ${ }^{2} \oplus$ \\ ${ }^{1}$ Department of Emergency, Faculty of Medicine, Yozgat Bozok University, Yozgat, Turkey \\ ${ }^{2}$ Department of Emergency Medicine, Faculty of Medicine, Cumhuriyet University, Sivas, Turkey
}

\begin{abstract}
INTRODUCTION: The main factors in the development of ST-segment elevation myocardial infarction (STEMI) are inflammatory processes and pathophysiological changes due to oxidant stress. The aim of this study is to evaluate the association of prevalent STEMI with markers of inflammatory and oxidative stress. The platelet to monocyte ratio (PMR) was evaluated as a new hematological inflammatory marker.
\end{abstract}

MATERIAL AND METHODS: The study is a retrospective observational study conducted in the emergency department between January 2018 and January 2019. In the study, all patients who were diagnosed with STEMI were considered in the study group. Evaluations of inflammatory and oxidant stress markers, PMR value of STEMI patients were performed.

RESULTS: Neutrophil to lymphocyte ratio (NLR) value of 5.63 (3.35-7.84), Monocyte count to high-density lipoprotein cholesterol ratio (MHR) value of 16.10 (12.73-19.52), gamma-glutamyl transferase (GGT) value of 31.00 (21-39) and CRP value of 5.10 (2.77-9.34) were significantly higher in STEMI cases $(p<0.005)$. The lymphocyte to monocyte ratio (LMR) value of 2.57 (2.00-3.61) and the PMR value of $24.52 \pm 7.60$ in STEMI cases were significantly lower $(p<0.001, p=0.014)$.

CONCLUSIONS: In STEMI patients NLR, GGT, and MHR rates increase significantly and LMR decreases. PMR values were also lower in STEMI cases. This shows us that PMR is a new determinant that can be used in inflammatory events. However, none of these inflammatory markers and oxidant stress markers can be used as diagnostic tests, rather they should be considered as surrogate markers for disease.

KEY WORDS: STEMI, NLR, LMR, PMR, inflammation, oxidative stress

Disaster Emerg Med J 2020; 5(4)

\section{INTRODUCTION}

ST-segment elevation myocardial infarction (STEMI) manifests as acute thrombotic coronary artery occlusion caused by atherosclerotic plaque rupture, plaque erosion or calcific plaque and is the leading cause of death worldwide $[1,2]$. The main factors in the development of this condition are inflammatory processes and pathophysiological changes due to oxidant stress [3].
Many complete blood count (CBC) parameters are useful predictive markers in cardiovascular events [4]. The increase in the neutrophil to lymphocyte ratio (NLR), the increase in monocyte count, the decrease in lymphocyte count and as a result the decrease in the lymphocyte to monocyte ratio (LMR), are considered indicative hematological biomarkers of inflammatory processes. At the same time, these 
markers are accepted as predictors both of the severity of the disease and the prognosis in coronary procedures for atherosclerotic diseases [5]. Gamma-glutamyl transferase (GGT) plays a role in the transport of glutathione, which in turn plays a role in intracellular antioxidant mechanisms. GGT levels increase in the case of an acute coronary syndrome such as atherosclerosis induced by oxidant stress [6]. High-density lipoprotein cholesterol (HDL-C) has a protective role against inflammation, oxidant stress and thrombus development. Monocytes are known to play a role in the development of atherosclerotic plaque [7]. Monocyte count to high-density lipoprotein cholesterol ratio (MHR) has been reported to increase in serious cardiovascular events as a result of the development of atherosclerosis [8].

The aim of this study was to evaluate the STEMI status in which inflammation and oxidant stress play a role together with factors such as NLR (which is an inflammatory marker) and GGT and MHR (which are oxidant stress markers). In addition, the platelet to monocyte ratio (PMR) was evaluated as a new hematological inflammatory marker in STEMI patients.

\section{MATERIAL AND METHODS Study design and population}

The study is a retrospective observational study conducted in the emergency department of Bozok University Faculty of Medicine between January 2018 and January 2019. The methodology of the study was in accordance with the protocol approved by the University Local Ethics Committee.

All patients who were admitted to the emergency department with chest pain and were diagnosed with STEMI or referred from the external center with the diagnosis of STEMI and whose blood was taken on arrival were considered for the study. All data were obtained by examining patients' files, electronic records in the hospital information system and angiography reports. STEMI was diagnosed by the presence of ST-segment elevation in at least two consecutive leads in electrocardiography (ECG), which was taken from patients with persistent chest pain or other suggestive symptoms of ischemia. STEMI diagnoses were confirmed by angio reports. There were 134 patients admitted with STEMI during the study period. The following patients $(n=48)$ were excluded from the study: those under the age of 18 , patients that had consumed alcohol, those with chronic liver function impairment or chronic liver disease (both of which can affect the GGT levels), patients with deep vein thrombosis, peripheral arterial disease, diabetes mellitus, metabolic syndrome, heart failure, rheumatic diseases, inflammatory bowel diseases, thyroid diseases and inflammatory diseases (which affect hematological inflammatory parameters), sufferers of familial hypercholesterolemia (which affects the $\mathrm{HDL}-\mathrm{C}$ ), patients receiving anticoagulant and antiaggregant treatment (which may cause a change in platelet counts) and patients with inadequate data. The remaining STEMI patients [ $\mathrm{n}=86: 28$ (32.6\%) female; 58 (76.4\%) male]. Healthy volunteers were included in the study as the control group, $[\mathrm{n}=82$ : 37 (44.6\%) female; 45 (54.2\%) male].

The following blood hematological parameters were examined from the first blood tests: hemoglobin $(\mathrm{Hb})$, hematocrit $(\mathrm{Htc})$, leucocyte, monocyte, neutrophil, red blood cell distribution width (RDW), mean platelet volume (MPV), mean corpuscular hemoglobin concentration (MCHC), platelet (PIt). GGT levels and lipid panels were also examined from the first blood samples. In addition, evaluations of NLR, LMR, platelet to lymphocyte ratio (PLR), PMR, MHR values of STEMI patients were performed.

\section{Laboratory analysis}

Tubes containing EDTA (Ethylenedinitrile-tetraacetic acid) were used for complete blood count (CBC) from all four groups. In order to prevent errors in platelet and other hematological parameters, samples were studied in the biochemistry laboratory with an XN-1000 hematology analyzer (Sysmex Corporation, Kobe, Japan) within the first hour. Serum gel tubes were used for biochemical analysis before centrifugation. Measurements of GGT, C-reactive protein (CRP), HDL-C were performed on a Cobas 6000 analyzer (Roche Diagnostics, Mannheim, Germany).

\section{Statistical analysis}

IBM SPSS Statistics for Windows version 23.0 (IBM, Armonk, NY, USA) was used for statistical analysis. The Kolmogorov-Smirnov test was used as a test for normality of continuous data and the results were expressed as the median with 25th and 75th percentiles. A non-parametric Mann-Whitney $U$ test was used to compare the medians between the two groups. ROC (receiver operating characteristic) analysis was performed to evaluate NLR, MHR, PLR, $L M R$ and PMR for predicting disease status. The area 
under the curve and the cut-off value were calculated for each value. Sensitivity, selectivity, positive predictive value and negative predictive value were calculated to evaluate the diagnostic test performance of each score. The statistical significance level was accepted as $p<0.05$.

\section{RESULTS}

Our study investigated the two groups: STEMI patients and the control group. The mean age of the STEMI group $(n=86)$ was $61 \pm 13$ years and the mean age of the control group $(n=82)$ was $62 \pm 12$ years. In the STEMI group, 43 (50\%) patients had inferior $\mathrm{Ml}, 30$ (34.9\%) had anterior $\mathrm{Ml}$, $1(1.2 \%)$ had lateral $\mathrm{MI}, 3(3.5 \%)$ had posterior $\mathrm{MI}$, $9(10.5 \%)$ had multiple vascular involvement MI.

\section{Comparison of the groups}

Table 1 shows the comparison of demographic data and laboratory parameters of the patient and control groups. NLR value of 5.63 (3.35-7.84), MHR value of 16.10 (12.73-19.52), GGT value of 31.00 (21-39) and CRP value of 5.10 (2.77-9.34) were significantly higher in STEMI cases compared to the control group $(p<00.5)$. The LMR value of $2.57(2.00-3.61)$ and the PMR value of $24.52 \pm 7.60$ in STEMI cases were significantly lower compared with the control group $(p<0.001, p=0.014$ ) (Fig. 1). When Hb, HCT, MCHC, PLT, HDL-C and PLR values were examined, no statistically significant difference was found between the two groups $(p>0.05)$.

\section{ROC analysis}

Table 2 shows the sensitivity, specificity, positive predictive values, negative predictive values and area under curve values of the patients and the control group according to ROC analysis. In the analysis, high GGT, MHR and NLR were found to be significant in favor of the disease $(p<0.001)$, whereas low LMR and PMR were found to be significant in favor of the disease $(p<0.001, p=0.014)$. In Figure 2, ROC analysis graphs of GGT, NLR, MHR, PLR, $L M R$ and PMR values are given.

\section{DISCUSSION}

Because both inflammatory processes and oxidant stress play an important role in the pathophysiology of acute coronary syndrome, blood parameters associated with these two conditions were examined together in this study. While NLR, GGT and MHR

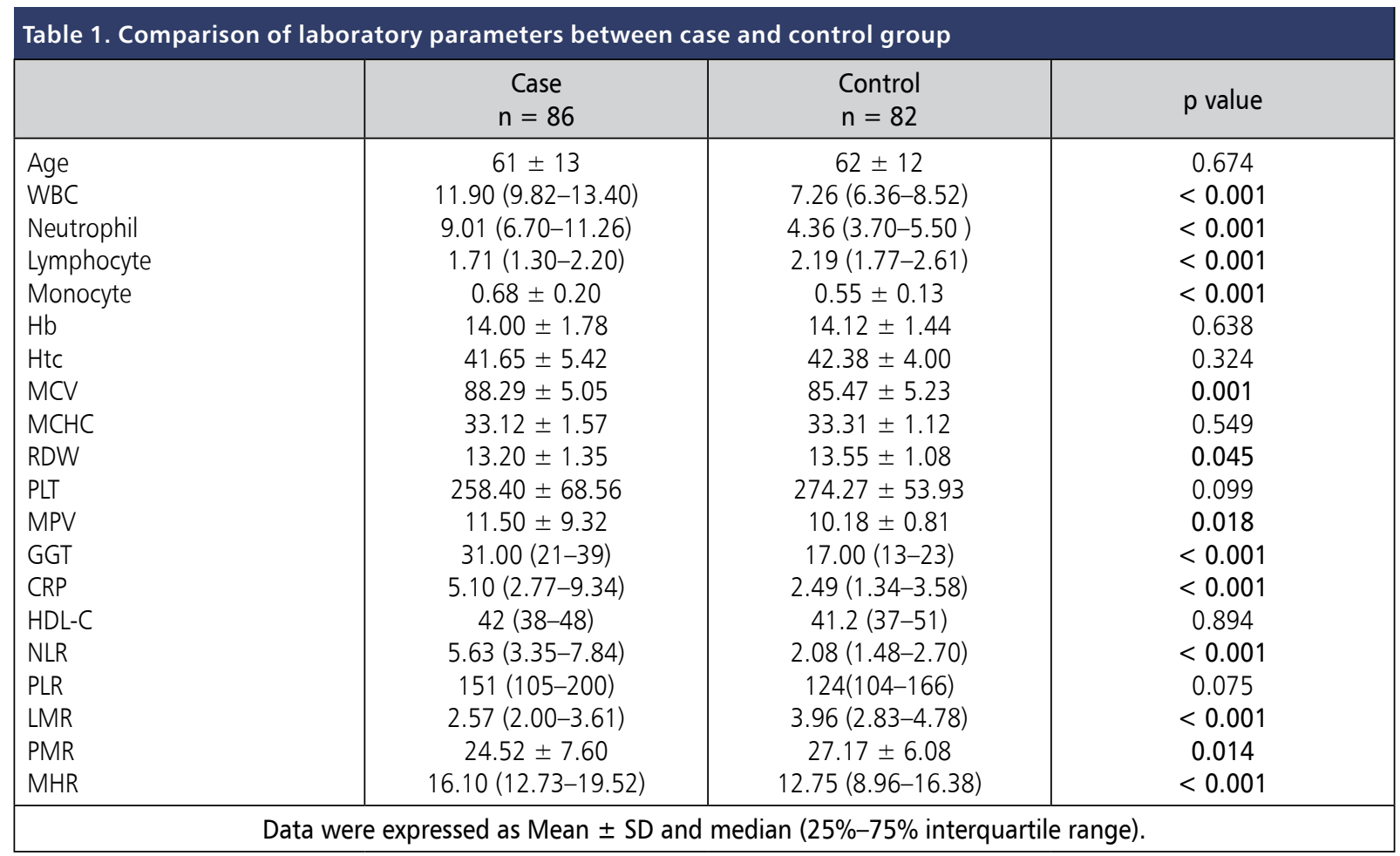

CRP — C-reactive protein; GGT — gamma-glutamyltransferase; Hb —-hemoglobin; HDL-C — High density lipoprotein cholesterol (HDL-C); Htc — hematocrit; LMR — lymphocyte to monocyte ratio; $\mathrm{MCHC}$ - mean corpuscular hemoglobin concentration; MCV — main corpuscular volume; MHR — monosite to HDL-C; MPV — mean platelet volume; NLR — neutrophil to lymphocyte ratio; PLR — platelet to lymphocyte ratio; PLT — platelet; PMR — platelet to lymphocyte ratio; RDW — Red blood cell distribution width 
A

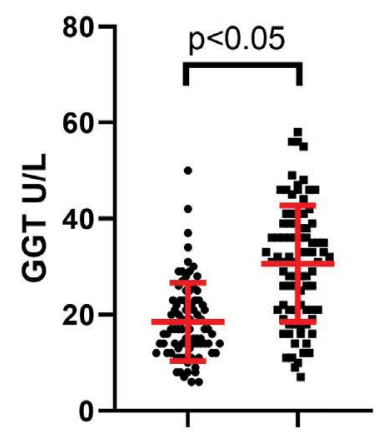

B
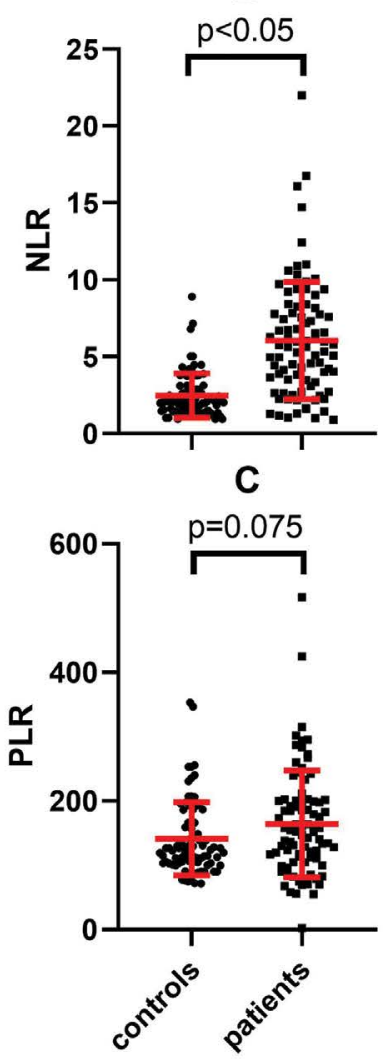

D
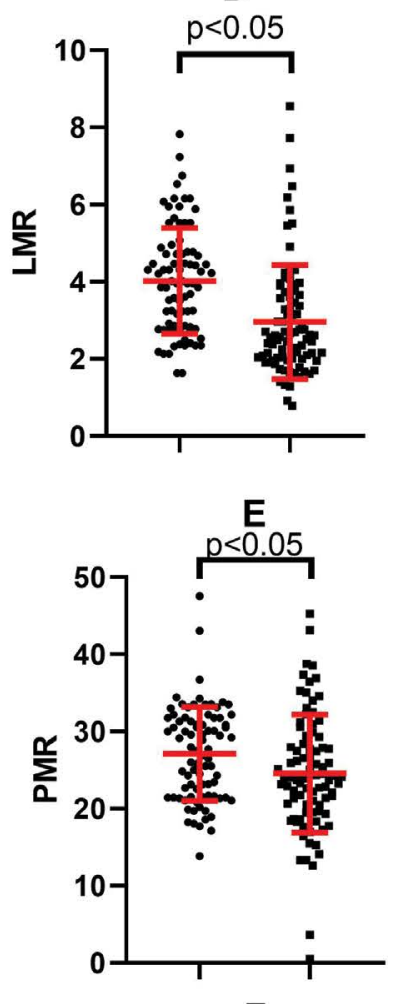

$\mathbf{F}$

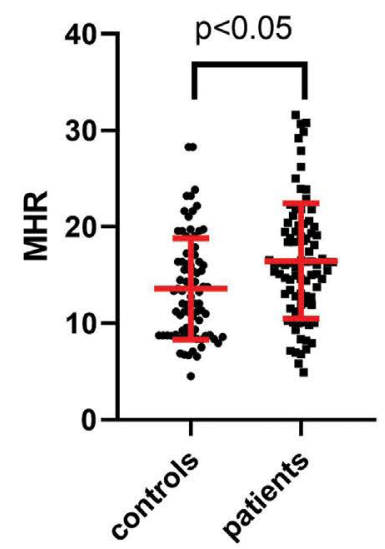

FIGURE 1. Relationship between parameters and disease

\begin{tabular}{|c|c|c|c|c|c|c|c|}
\hline \multirow{2}{*}{ Variables } & \multicolumn{5}{|c|}{ Statistical diagnostic measures } & \multicolumn{2}{|c|}{ ROC curve statistics } \\
\hline & Cutoff & SEN $(95 \% \mathrm{Cl})$ & SPE $(95 \% \mathrm{Cl})$ & PPV $(95 \% \mathrm{Cl})$ & NPV $(95 \% \mathrm{Cl})$ & AUC & $\mathrm{p}$ \\
\hline GGT & 17.5 & 0.839 & 0.568 & 83.9 & 56.8 & 0.793 & $<0.001$ \\
\hline NLR & 2.152 & 0.897 & 0.580 & 89.7 & 58.0 & 0.836 & $<0.001$ \\
\hline MHR & 14.370 & 0.667 & 0.605 & 66.7 & 60.5 & 0.642 & 0.001 \\
\hline PLR & & & & & & 0.580 & 0.075 \\
\hline LMR* & 2.722 & 0.840 & 0.586 & 41.4 & 84.0 & 0.747 & $<0.001$ \\
\hline PMR* & 19.692 & 0.914 & 0.253 & 74.7 & 8.6 & 0.610 & 0.014 \\
\hline
\end{tabular}

AUC — Area under curve; Cl — Confidence interval; GGT — gama-glutamyl transferase; LMR — lymphocyte to monocyte ratio; MHR — monosite to HDL-C; NLR — neutrophil to lymphocyte ratio; NPV — Negative predictive value; PLR — platelet to lymphocyte ratio; PMR — platelet to lymphocyte ratio; PPV — Positive predictive value; ROC — Receiver operating characteristic; SEN — Sensitivity; SPE — Specificity

${ }^{*}$ a decrease in the value causes the disease 
GGT

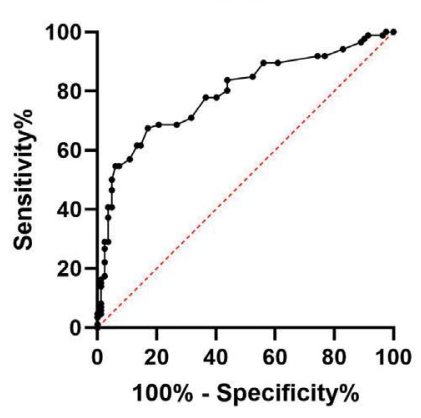

NLR

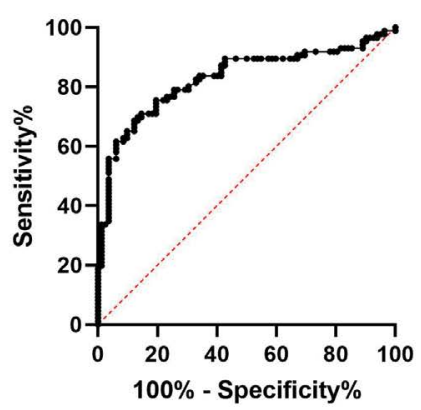

PLR

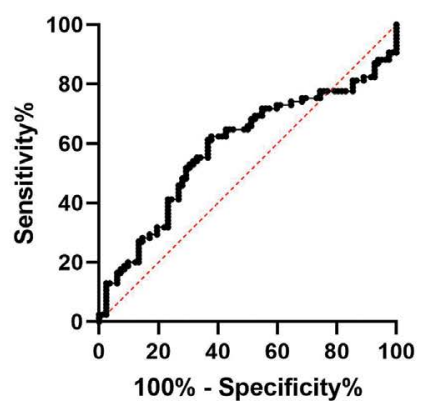

LMR

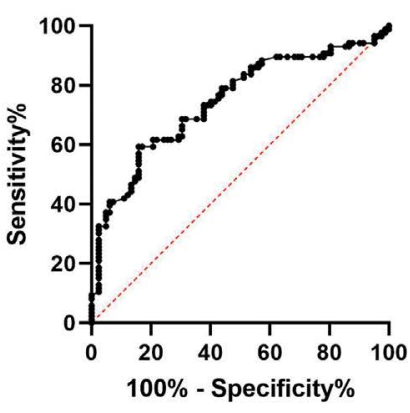

PMR

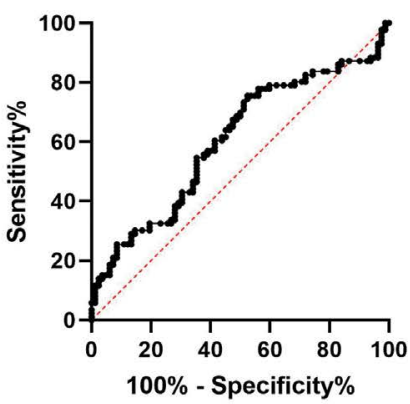

MHR

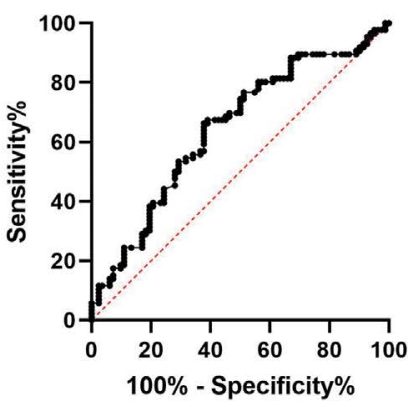

FIGURE 2. Receiver operating characteristic curves of inflammatory parameters according to the presence of ST-elevation myocardial infarction

rates were found to be significant in terms of STEMI disease, low LMR and PMR values were also significant in terms of disease. It has been determined that MHR cannot be used as a marker of disease, but it can be useful in terms of prognosis and guidance. In addition, low levels of PMR can be used as an inflammatory marker in STEMI cases.

NLR, PLR and LMR values are used as markers that determine the inflammatory response in many diseases including in cancer cases such as CRP $[9,10]$. In addition to elevated systemic inflammatory response, high levels of NLR has been reported to be an independent risk factor for increased mortality and long-term poor prognosis in both acute coronary syndrome and STEMI patients [11]. In our study, high levels of NLR support this information.
In the evaluations for PLR, both platelet increase and lymphocyte decrease have been shown to be a poor prognosis predictor for coronary events. Azap et al. [10] found that PLR increase is a predictor of longterm mortality in non-STEMI cases. In our study, although the PLR rates in STEMI cases were higher than the control group, no statistical significance was found. It has been emphasized in many studies that LMR, which is expected to decrease in cases of malignancy and viral infection, is a predictor of coronary slow flow in patients with coronary artery disease and has a negative correlation with both CRP and NLR [12-14]. Another study showed that increased platelet and monocyte activity was found to be the predictor of atherosclerosis in hemodialysis patients and peritoneal dialysis patients [15]. In 
a study on patients with chronic obstructive pulmonary disease (COPD), platelet-monosyte interaction was found to have increased [16] but to our knowledge, PMR has not been considered as an inflammatory marker in any study including coronary artery disease. In patients with coronary artery disease, no change was reported in platelet levels unless antiaggregant treatment was used, but changes in platelet shape and volumetric measurements, such as MPV, were detected [17]. In our study, patients receiving anticoagulant and antiaggregant treatment were excluded and no difference was found between STEMI cases and the control group in terms of platelet measurements. The ratio of platelets to monocytes as a result of inflammatory processes was found to be low in STEMI cases.

It has been shown that the level of GGT increases both in hepatobiliary system pathology and with alcohol use and is accepted as a marker for these conditions $[18,19]$. In addition to being an oxidant stress biomarker [6], GGT was also found to be active in the oxidation of low-density lipoprotein (LDL) and the development of atheroma plaques in the coronary arteries [20]. Valjevac et al. [21] found a positive correlation between GGT and CRP in patients with the acute coronary syndrome. The GGT elevation in STEMI cases detected in our study confirms this finding.

Monocytes, macrophages and dendritic cell precursors play a key role in the development of atherosclerosis. Circulating monocytes in patients with cardiovascular disease have a high level of surface receptors in the inflammatory response. Monocytes present in the inflammation area form a complex with oxidized LDL. This complex is described as a dendritic cell or inflammatory macrophage. These cells cause atherosclerosis by forming oily streaks or foam cells [22]. HDL-C has anti-inflammatory and antioxidant effects on endothelial functions, in particular, by interfering with or even reversing monocyte activation and adhesion [7]. A literature search in the light of this information showed that MHR is an independent risk factor for five-year mortality in STEMI patients [8]. Demir et al. [23] found that MHR was elevated in patients with metabolic syndrome. This increase is due to decreasing HDL-C levels in metabolic syndrome. The antioxidant activity of HDL-C is well known [24]. We found that since there was no significant difference between HDL-C levels in STEMI patients and the control group, the elevation of MHR was independent of antioxidant mechanisms and due to the increase in monocytes as an inflammatory marker.

There are some limitations to our study. Firstly, it was a single-center study and therefore we had a limited number of patients. Secondly, our study was retrospective and based on files and electronic records. Multi-center studies with larger patient groups are needed.

\section{CONCLUSIONS}

Previous studies have investigated hematological inflammatory markers and oxidant stress markers separately in STEMI cases. However, to the best of our knowledge, there has not been a study that examines all of these markers together. In STEMI patients NLR, GGT, and MHR rates increase significantly and LMR decreases. Cut off values for GGT and NLR show high sensitivity for the disease. In our study, PMR values were also lower in STEMI cases. This shows us that PMR is a new determinant that can be used in inflammatory events. However, none of these inflammatory markers and oxidant stress markers can be used as diagnostic tests, rather they should be considered as surrogate markers for disease.

Conflict of interest: The authors have no conflict of interest to declare.

Declaration of interest: All authors disclose any financial and personal relationships with other people or organizations that could inappropriately influence their work.

Submission declaration: All authors declare that this paper is not published or under consideration for publishing elsewhere.

Journal policies: All authors reviewed in detail the journal policies.

Ethical conduct of research: The authors have obtained approval from the review board of the Bozok University Faculty of Medicine, decision number 2017-KAEK-189_2019.11.13_06.

\section{REFERENCES}

1. Choudhury T, West NEj, El-Omar M. ST elevation myocardial infarction. Clin Med (Lond). 2016; 16(3): 277-282, doi: 10.7861/clinmedicine.16-3-277, indexed in Pubmed: 27251920. 
2. Afari ME, Bhat T. Neutrophil to lymphocyte ratio (NLR) and cardiovascular diseases: an update. Expert Rev Cardiovasc Ther. 2016; 14(5): 573-577, doi: 10.1586/14779072.2016.1154788, indexed in Pubmed: 26878164.

3. Heitzer T, Schlinzig T, Krohn K, et al. Endothelial dysfunction, oxidative stress, and risk of cardiovascular events in patients with coronary artery disease. Circulation. 2001; 104(22): 2673-2678, doi: 10.1161/ hc4601.099485, indexed in Pubmed: 11723017.

4. Gunes H, Saritas A, Cangur S, et al. Complete Blood Count Parameters for Prediction of non-ST Segment Elevation Myocardial Infarction. J Coll Physicians Surg Pak. 2019; 29(8): 710-714, doi: 10.29271/ jcpsp.2019.08.710, indexed in Pubmed: 31358088.

5. Kiris T, Çelik A, Variş E, et al. Association of Lymphocyte-to-Monocyte Ratio With the Mortality in Patients With ST-Elevation Myocardial Infarction Who Underwent Primary Percutaneous Coronary Intervention. Angiology. 2017; 68(8): 707-715, doi: 10.1177/0003319716685480, indexed in Pubmed: 28056530.

6. Gul M, Uyarel H, Ergelen $M$, et al. The relationship between -glutamyl transferase levels and the clinical outcomes in patients with ST-segment elevation myocardial infarction undergoing primary percutaneous coronary intervention. Coron Artery Dis. 2013; 24(4): 272-278, doi: 10.1097/MCA.0b013e328360d131, indexed in Pubmed: 23542158.

7. Arısoy A, Altunkaş F, Karaman K, et al. Association of the Monocyte to HDL Cholesterol Ratio With Thrombus Burden in Patients With ST-Segment Elevation Myocardial Infarction. Clin Appl Thromb Hemost. 2017; 23(8): 992-997, doi: 10.1177/1076029616663850, indexed in Pubmed: 27534422.

8. Açıgöz SK, Açıkgöz E, Şensoy $B$, et al. Monocyte to high-density lipoprotein cholesterol ratio is predictive of in-hospital and five-year mortality in ST-segment elevation myocardial infarction. Cardiol J. 2016; 23(5): 505-512, doi: 10.5603/CJ.a2016.0026, indexed in Pubmed: 27296159.

9. Lee SM, Russell A, Hellawell G. Predictive value of pretreatment inflammation-based prognostic scores (neutrophil-to-lymphocyte ratio, platelet-to-lymphocyte ratio, and lymphocyte-to-monocyte ratio) for invasive bladder carcinoma. Korean J Urol. 2015; 56(11): 749-755, doi: 10.4111/kju.2015.56.11.749, indexed in Pubmed: 26568792.

10. Azab B, Shah N, Akerman M, et al. Value of platelet/lymphocyte ratio as a predictor of all-cause mortality after non-ST-elevation myocardial infarction. J Thromb Thrombolysis. 2012; 34(3): 326-334, doi: 10.1007/s11239-012-0718-6, indexed in Pubmed: 22466812.

11. Akpek M, Kaya MG, Lam YY, et al. Relation of neutrophil/lymphocyte ratio to coronary flow to in-hospital major adverse cardiac events in patients with ST-elevated myocardial infarction undergoing primary coronary intervention. Am J Cardiol. 2012; 110(5): 621-627, doi: 10.1016/j.amjcard.2012.04.041, indexed in Pubmed: 22608360.

12. Yayla Ç, Akboğa MK, Gayretli Yayla K, et al. A novel marker of inflammation in patients with slow coronary flow: lymphocyte-to-monocyte ratio. Biomark Med. 2016; 10(5): 485-493, doi: 10.2217/bmm-20160022, indexed in Pubmed: 27089433.

13. Stotz $M$, Pichler $M$, Absenger $G$, et al. The preoperative lymphocyte to monocyte ratio predicts clinical outcome in patients with stage III colon cancer. Br J Cancer. 2014; 110(2): 435-440, doi: 10.1038/ bjc.2013.785, indexed in Pubmed: 24357796.

14. Merekoulias G, Alexopoulos EC, Belezos T, et al. Lymphocyte to monocyte ratio as a screening tool for influenza. PLoS Curr. 2010; 2: RRN1154, doi: 10.1371/currents.rrn1154, indexed in Pubmed: 20383263.

15. Stach K, Karb S, Akin I, et al. Elevation of Platelet and Monocyte Activity Markers of Atherosclerosis in Haemodialysis Patients Compared to Peritoneal Dialysis Patients. Mediators Inflamm. 2017; 2017: 8506072 , doi: 10.1155/2017/8506072, indexed in Pubmed: 28769539.

16. Aleva FE, Temba $G$, de Mast $Q$, et al. Increased Platelet-Monocyte Interaction in Stable COPD in the Absence of Platelet Hyper-Reactivity. Respiration. 2018; 95(1): 35-43, doi: 10.1159/000480457, indexed in Pubmed: 29020675.

17. Khode V, Sindhur J, Kanbur D, et al. Mean platelet volume and other platelet volume indices in patients with stable coronary artery disease and acute myocardial infarction: A case control study. J Cardiovasc Dis Res. 2012; 3(4): 272-275, doi: 10.4103/0975-3583.102694, indexed in Pubmed: 23233769.

18. Jiang $S$, Jiang $D$, Tao $Y$. Role of gamma-glutamyltransferase in cardiovascular diseases. Exp Clin Cardiol. 2013;18(1):53-6. PMID: 24294039; PMCID. : PMC3716505.

19. Kim JG, Chang K, Choo EHo, et al. Serum gamma-glutamyl transferase is a predictor of mortality in patients with acute myocardial infarction. Medicine (Baltimore). 2018; 97(29): e11393, doi: 10.1097/ MD.0000000000011393, indexed in Pubmed: 30024510.

20. Emdin M, Passino C, Michelassi C, et al. Prognostic value of serum gamma-glutamyl transferase activity after myocardial infarction. Eur Heart J. 2001; 22(19): 1802-1807, doi: 10.1053/euhj.2001.2807, indexed in Pubmed: 11549302.

21. Valjevac A, Rebic D, Hamzic-Mehmedbasic $A$, et al. The value of gamma glutamyltransferase in predicting myocardial infarction in patients with acute coronary syndrome. Future Cardiol. 2018; 14(1): 37-45, doi: 10.2217/fca-2017-0033, indexed in Pubmed: 29199860.

22. Ley K, Miller YI, HedrickCC. Monocyte and macrophage dynamics during atherogenesis. Arterioscler Thromb Vasc Biol. 2011; 31(7): 1506-1516, doi: 10.1161/ATVBAHA.110.221127, indexed in Pubmed: 21677293.

23. Vahit D, Akboga MK, Samet $Y$, et al. Assessment of monocyte to high density lipoprotein cholesterol ratio and lymphocyte-to-monocyte ratio in patients with metabolic syndrome. Biomark Med. 2017; 11(7): 535540, doi: 10.2217/bmm-2016-0380, indexed in Pubmed: 28685581.

24. Escolà-Gil JC, Rotllan N, Julve J, et al. In vivo macrophage-specific RCT and antioxidant and antiinflammatory HDL activity measurements: New tools for predicting HDL atheroprotection. Atherosclerosis. 2009; 206(2): 321-327, doi: 10.1016/j.atherosclerosis.2008.12.044, indexed in Pubmed: 19362310. 